\title{
Microwave-Assisted Evaporative Crystallization of Niflumic Acid for Particle Size Reduction
}

\author{
Norbert Radacsi, ${ }^{\dagger}$ Joop H. ter Horst, ${ }^{\dagger}$ and Georgios D. Stefanidis ${ }^{\dagger}{ }^{\dagger}$ \\ ${ }^{\dagger}$ Intensified Reaction \& Separation Systems, Process \& Energy Laboratory, Delft University of Technology, Leeghwaterstraat 44, 2628 \\ CA, Delft, The Netherlands
}

\section{Supporting Information}

ABSTRACT: Microwave irradiation can significantly speed up solvent evaporation in crystallization processes, resulting in rapid crystallization and reduced particle size. A single-mode microwave setup was used for evaporative crystallization of the model pharmaceutical compound, niflumic acid. Production of crystals by microwave irradiation offers a novel way to particles for drug formulation solving formulation problems of drugs with low solubility and permeability in class II of the Biopharmaceutics Classification System. In this work, niflumic acid was produced using microwave irradiation and conventional heating. The

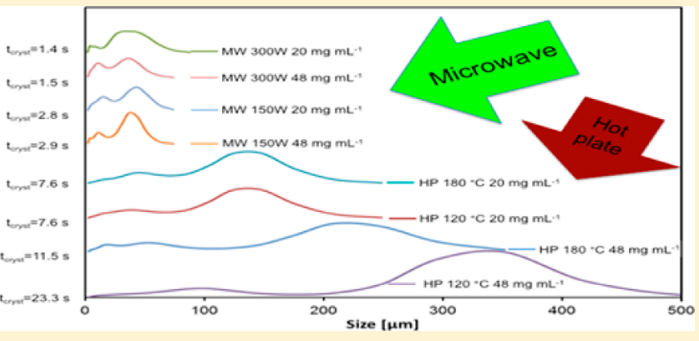
final product size, as measured by a particle size analyzer, was reduced when increasing the evaporation rate using microwave irradiation. X-ray diffraction proved that the crystal form after microwaveassisted crystallization remained unchanged.

S olving bioavailability problems of new active pharmaceutical ingredients is a major challenge for the pharmaceutical industry, since nearly half of the new active substances being identified in high-throughput screening are either insoluble or poorly soluble in water. ${ }^{1-3}$ As the crystallization method determines the product properties, it is possible to increase the dissolution rate and reach higher drug bioavailability by selecting proper crystallization conditions. ${ }^{4,5}$ Evaporative crystallization is widely applied in several industrial processes, including the pharmaceutical industry.

The effect of microwave heating on the evaporative crystallization of niflumic acid (NIF) is investigated in this study. NIF is an important active pharmaceutical ingredient, with anti-inflammatory activity accompanied by an analgesic effect. ${ }^{6}$ In accordance with the Biopharmaceutics Classification System (BCS), NIF belongs to class II, a practically waterinsoluble $\left(26 \mu \mathrm{g} \mathrm{mL}^{-1}\right.$ at $\left.25{ }^{\circ} \mathrm{C}\right)$, lipophilic, and highly permeable compound. ${ }^{7}$ To achieve optimal pharmacodynamic properties such as a rapid onset of the drug effect, fast dissolution is essential for this type of drug.

Microwave heating is fundamentally different from conventional (conductive) heating. In particular, microwaves offer rapid and volumetric heating, without the need of heat transfer surfaces or heat transfer fluids. In addition, as microwaves couple directly with the (polar) molecules of the solution, heat transfer stops immediately when the magnetron is turned off, thus minimizing thermal inertia. Over the past 30 years, many interesting phenomena have been observed and reported in studies on microwave-enhanced chemistry. ${ }^{8}$ As regards crystallization, Pinard and Aslan ${ }^{9}$ reported that microwaves enable crystallization of glycine from aqueous solutions onto silver nanostructures coated on glass surface in seconds. The silver nanoparticles were meant to serve as selective nucleation sites and as a microwave-transparent medium for the creation of thermal gradient between the warmer solution and the silver nanoparticles. The thermal gradient afforded for the mass transfer and assembly of glycine molecules onto the nanoparticles. Contrary to Pinard and Aslan, we focus on the effect of microwaves on the solvent evaporation rate and how this affects crystal size.

Clear undersaturated solutions of NIF in ethanol at two different concentrations $\left(20 \mathrm{mg} \mathrm{mL}^{-1}\right.$ and $\left.48 \mathrm{mg} \mathrm{mL}^{-1}\right)$ were prepared, and a $5 \mathrm{~mL}$ volume of solution was injected into a quartz Petri dish. The Petri dish was placed in the middle of the cavity of the single-mode microwave setup (Figure 1) for the microwave experiments or on top of a hot plate in the case of conventional heating experiments. In total, eight different experimental conditions were investigated.

In the event of microwave heating, heat transfer does not depend on the thermal conductivity, which is low for conventional solvents, like water, methanol, or ethanol. ${ }^{10}$ Rather, it depends on the dielectric properties of the solution. Ethanol has favorable dielectric properties for microwave heating, as it has a very high loss tangent $(\tan \delta)$, a measure of inherent dissipation of electromagnetic energy of the material $(0.941$ at $2.45 \mathrm{GHz}){ }^{11}$

When microwave heating is used for solvent evaporation, the $5 \mathrm{~mL}$ solution starts boiling within $4 \mathrm{~s}$ with maximum microwave power $(300 \mathrm{~W})$ (see Figure S1 of the Supporting Information) and within $8 \mathrm{~s}$ at half of the maximum microwave power $(150 \mathrm{~W})$. The solvent was completely evaporated within

Received: July 18, 2013

Revised: August 24, 2013

Published: August 28, 2013 


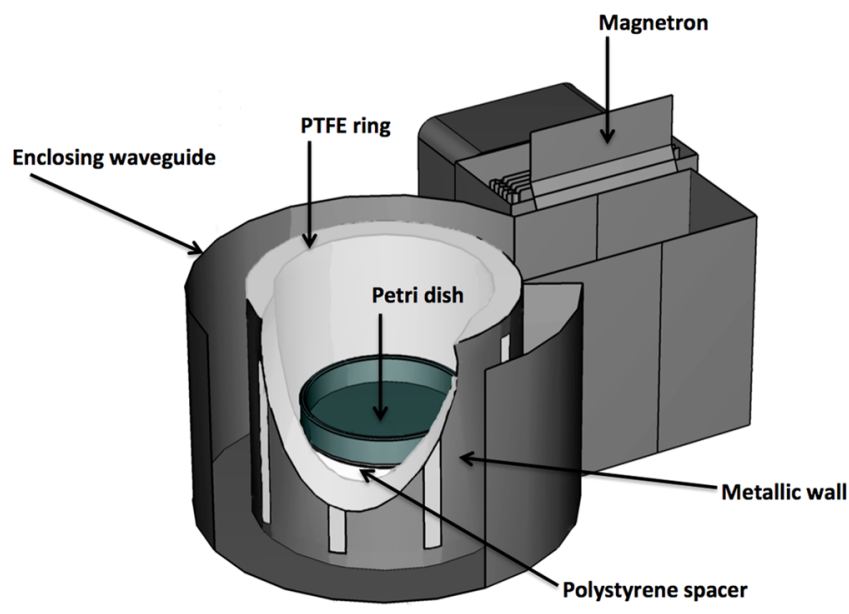

Figure 1. Cutaway view of the used microwave setup showing the position of the quartz Petri dish and the main parts of the setup.

$21 \mathrm{~s}$ at $300 \mathrm{~W}$ (see the movie of the Supporting Information) and within $43 \mathrm{~s}$ at $150 \mathrm{~W}$ (Table 1 ).

Table 1. Time Needed for Complete Evaporation $\left(t_{\text {evap }}\right)$ of 5 mL NIF Dissolved in Ethanol; Crystallization Time $\left(t_{\text {cryst }}\right)$, Mean Diameter over Volume (MV) and Standard Deviation (SD) for the Two Initial Solvent Concentrations (c) Applied, Under Microwave and Hot Plate Heating

\begin{tabular}{cccccc}
$\begin{array}{c}c(\mathrm{mg} \\
\text { method }\end{array}$ & $\begin{array}{c}t_{\text {evap }} \\
\left.\mathrm{mL}^{-1}\right)\end{array}$ & $\begin{array}{c}t_{\text {cryst }} \\
(\mathrm{s})\end{array}$ & $\begin{array}{c}\mathrm{MV} \\
(\mu \mathrm{m})\end{array}$ & $\begin{array}{c}\mathrm{SD} \\
(\mu \mathrm{m})\end{array}$ \\
microwave $300 \mathrm{~W}$ & 20 & 20 & 1.4 & 25 & 17 \\
microwave $150 \mathrm{~W}$ & 48 & 21 & 1.5 & 19 & 13.9 \\
& 20 & 43 & 2.8 & 23 & 15.9 \\
hot plate $180{ }^{\circ} \mathrm{C}$ & 48 & 41 & 2.9 & 23 & 14.9 \\
& 20 & 78 & 7.6 & 81 & 52.6 \\
hot plate $120{ }^{\circ} \mathrm{C}$ & 48 & 70 & 11.5 & 110 & 91 \\
& 20 & 166 & 7.6 & 79 & 55 \\
& 48 & 167 & 23.3 & 209 & 133 \\
\hline
\end{tabular}

Using conventional heating resulted in significantly larger times for complete solvent evaporation, even though the solution started to boil immediately after injection. More specifically, at $120{ }^{\circ} \mathrm{C}$ and $180{ }^{\circ} \mathrm{C}$ Petri dish temperatures, at least 166 and $70 \mathrm{~s}$, respectively, were needed for complete solvent evaporation of the $20 \mathrm{mg} \mathrm{mL}^{-1}$ solutions (Table 1).

In the evaporation process (either heat conduction- or microwave-assisted), the time window between the time at which the solution becomes supersaturated and the time of complete solvent evaporation is defined as the crystallization time. During this crystallization time at some point nucleation and subsequently crystal growth occurs, the rate of both then determine the final crystal size distribution.

Two process parameters were identified to influence crystallization time. First is the heat transfer rate, which can be regulated by the applied microwave power or the Petri dish surface temperature in the event of conductive (hot plate) heating. Second is the initial solution concentration. When the initial concentration is increased, supersaturation is reached earlier, while the time needed for solvent evaporation remains the same, which results in a larger crystallization time. The crystallization time $t_{\text {cryst }}$ for all process conditions tested, together with the relevant product size characterization values (mean volume diameter and standard deviation), are shown in
Table 1, while the experimental approach to obtain the crystallization times and the discussion of results follows in the next paragraphs.

During all the hot plate evaporative crystallization experiments and during the microwave irradiation experiment using maximum power and $20 \mathrm{mg} \mathrm{mL} \mathrm{m}^{-1}$ initial solution concentration, the total mass of the solution was monitored. It was found that the solvent evaporation rate was close to constant, resulting in a linear change of the cumulative evaporated solvent mass with time (see Figure S2 of the Supporting Information). From the solution mass, the mass fraction of the solute in the solution was calculated and plotted as a function of time (Figure 2). It was thus assumed that the solvent evaporation rate is also constant in the other experiments.

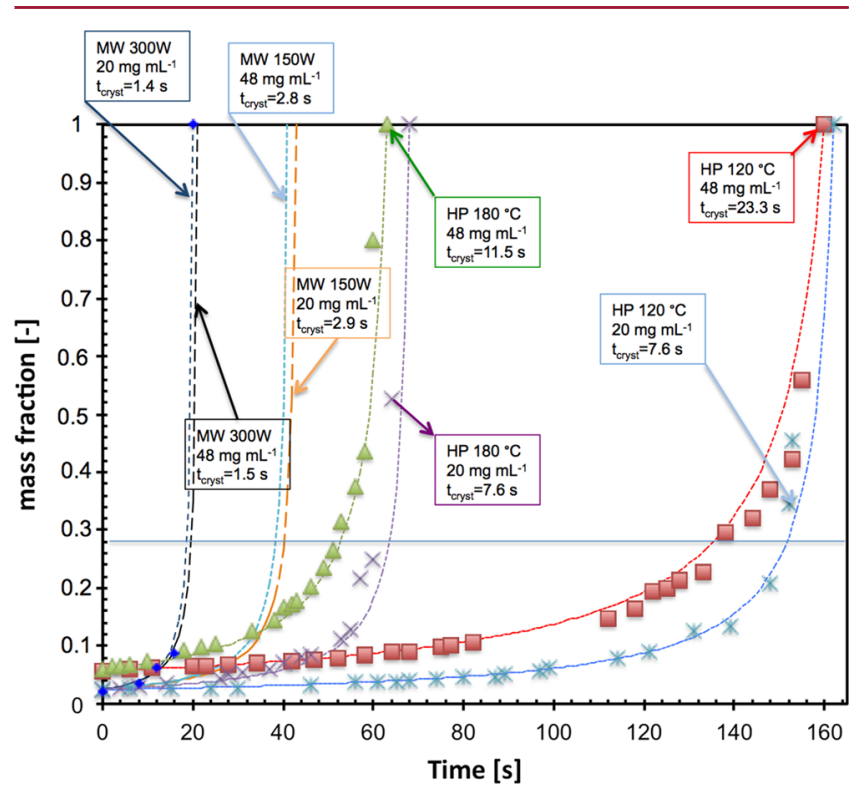

Figure 2. Mass fraction of solute in the solvent. MW stands for microwave-assisted crystallization, and HP refers to evaporative crystallization on top of a hot plate. The dashed lines represent the theoretical lines, assuming constant evaporation rate.

In order to determine the crystallization times, the solubility of NIF in ethanol at different temperatures needs to be known. The solubility of NIF in ethanol (see Figure S3 of the Supporting Information) was determined using saturation temperature measurements of differently concentrated samples. NIF has a solubility of $56 \mathrm{mg} \mathrm{mL}^{-1}$ at room temperature $(25$ ${ }^{\circ} \mathrm{C}$ ), and an interpolated solubility of around $213 \mathrm{mg} \mathrm{mL}^{-1}$, equal to a mass fraction of 0.28 at the boiling point of ethanol $\left(78.3{ }^{\circ} \mathrm{C}\right)$.

It was found that microwave irradiation for evaporative crystallization results in very small crystallization times due to the high evaporation rates achieved; this implies high supersaturation ratios and thus high nucleation rates. As a result, the mean volume diameter (MV), determined by laser diffraction particle size analysis, was $25 \mu \mathrm{m}$ at maximum, in the microwave experiments. In the hot plate experiments, the crystal size was found to be significantly larger. In the case of 20 $\mathrm{mg} \mathrm{mL}{ }^{-1}$ solution concentration, crystal sizes of around $80 \mu \mathrm{m}$ were obtained, while in the case of $48 \mathrm{mg} \mathrm{mL}^{-1}$ initial solution concentration, even larger sizes were obtained with a minimum of $110 \mu \mathrm{m}$ diameter. The standard deviation is also significantly smaller for the products produced with use of microwaves than 
the products produced on the hot plate (see Table 1). Thus microwave-assisted evaporative crystallization results in small crystal size with narrow standard deviation. The crystal size distributions for each crystallization time are shown in Figure 3.

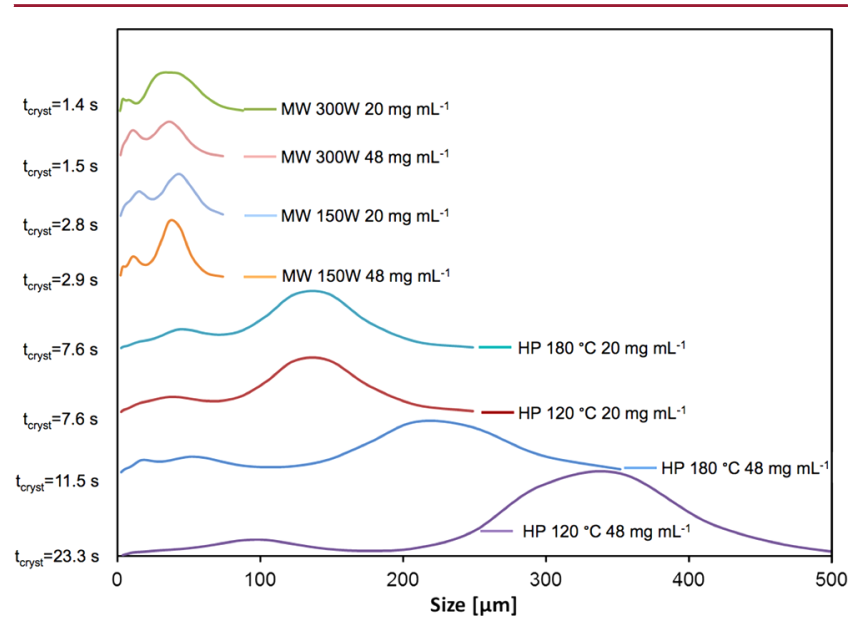

Figure 3. The crystal size distribution is increasing with the crystallization time.

Next to the larger needle-like crystals, small (1-3 $\mu \mathrm{m})$ crystals with compact shapes can be observed (see Figure S4 for SEM images of the Supporting Information). It can be seen that the crystal size distributions of the microwave products are bimodal, which is most probably due to the occurrence of several nucleation events originating from the nonuniformity of the electromagnetic field ${ }^{12-14}$ and the non-optimal constant evaporation rate during the experiment. At the end of the experiment, the high evaporation rate leads to extremely high supersaturation rates, and nucleation might dominate growth, leading to bimodal crystal size distributions consisting of larger particles nucleated earlier in the experiment and smaller particles that nucleated toward the end.

It was found that the final crystal size and the standard deviation increase approximately linearly with increasing crystallization time (Figure 4).

X-ray diffraction showed that neither the microwave irradiation nor the hot plate aided evaporative crystallization altered the crystal structure of NIF, as compared to that reported in literature ${ }^{15}$ (see Figure S5 of the Supporting Information).

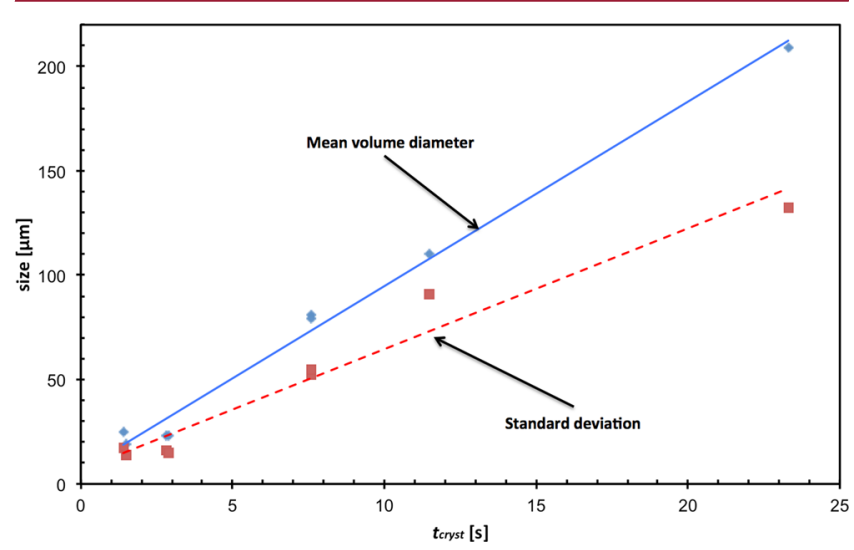

Figure 4. There is a linear correlation between the mean volume diameter and standard deviation with the crystallization time.
Microwave-assisted evaporative crystallization appears to be a promising method for crystal size reduction due to fast evaporation rates generating high supersaturations. It is possible to scale up microwave reactors toward industrial scale, using a promising new concept of coupling microwave energy to liquid solutions. In particular, the INTLI (internal transmission line) technology allows for effective microwave heating of liquid mixtures in up to $17 \mathrm{~L}$ stainless steel stirred batch reactors at present. $^{16-19}$

\section{ASSOCIATED CONTENT}

\section{Supporting Information}

The experimental details, the temperature profile for heating with microwaves, evaporation rate profiles, the solubility line of NIF in ethanol, a SEM image of the NIF crystals produced by microwave-assisted evaporation, XRPD patterns of NIF, and a movie of the microwave-assisted evaporation experiment. This material is available free of charge via the Internet at http:// pubs.acs.org.

\section{AUTHOR INFORMATION}

\section{Corresponding Author}

*E-mail: g.stefanidis@tudelft.nl. Tel: +31 152781447.

\section{Notes}

The authors declare no competing financial interest.

\section{ACKNOWLEDGMENTS}

The research leading to these results has received funding from the European Community's Seventh Framework Programme under Grant FP7-NMP-2012-309874. We would like to thank Guido Sturm for the help with the CEM Discover system, Peetikamol Kongsamai for measuring the solubility of niflumic acid in ethanol and Rita Ambrus for the SEM images.

\section{REFERENCES}

(1) Handbook of Industrial Crystallization; Myerson, A. S., Ed.; Butterworth-Heinemann Ltd.: Oxford, 1993.

(2) Amidon, G. L.; Löbenberg, R. Eur. J. Pharm. Biopharm. 2000, 50, 3-12.

(3) Patravale, V. B.; Date, A. A.; Kulkarni, R. M. J. Pharm. Pharmacol. 2004, 56, 827-840.

(4) Huang, L. F.; Tong, W. Q. Adv. Drug Delivery Rev. 2004, 56, $321-334$

(5) Shekunov, B. Y.; York, P. J. Cryst. Growth 2000, 211, 122-136.

(6) Insel, P. A. Analgesic-Antipyretics and Antiinflammatory Agents; Drugs Employed in the Treatment of Rheumatoid Arthritis and Gout. In Goodman and Gilman's The Pharmacological Basis of Therapeutics; Gilman, A. G., Rall, T. W., Nies, A. S., Taylor, P., Eds.; McGraw-Hill: Singapore, 1991; p 668.

(7) Houin, G.; Tremblay, D.; Bree, F.; Dufour, A.; Ledudal, P.; Tillement, J. P. Int. J. Clin. Pharmacol., Ther. Toxicol. 1983, 21, 130134.

(8) Kappe, O. C. Chem. Soc. Rev. 2008, 37, 1127-1139.

(9) Pinard, M. A.; Aslan, K. Cryst. Growth Des. 2010, 10, 4706-4709.

(10) Assael, M. J.; Charitidou, E.; Wakeham, W. A. Int. J. Thermophys. 1989, 10, 793-804.

(11) Metaxas, A. C. Foundations of Electroheat: A Unified Approach; Wiley: New York, 1996.

(12) Sturm, G. S. J.; Verweij, M. D.; Van Gerven, T.; Stankiewicz, A. I.; Stefanidis, G. D. Int. J. Heat Mass Transfer 2012, 55, 3800-3811.

(13) Sturm, G. S. J.; Verweij, M. D.; Van Gerven, T.; Stankiewicz, A. I.; Stefanidis, G. D. Int. J. Heat Mass Transfer 2013, 57, 375-388.

(14) Durka, T.; Stefanidis, G. D.; Van Gerven, T.; Stankiewicz, A. I. Meas. Sci. Technol. 2010, 21, 045108. 
(15) Krishna Murthy, H. M.; Vijayan, M. Acta Crystallogr. 1979, B35, 262-263.

(16) SAIREM. http://www.sairem.com/ (accessed August 23, 2013).

(17) Grange, A.; Jacomino, J.; Grandemenge, A.; Angdeure, G.; Mari, J. J.; Adeurieng, G. Device for Applying Electromagnetic Radiation to Reaction Medium, e.g. for Extracting Biomaterial, Has Loss Transmission Line to Transfer Controlled Energy Density Profile to Medium. Patent US 2011104793 Al, 2011.

(18) Grange, A.; Jacomino, J.; Grandemenge, A. Device for Electromagnetic Radiation Treatment of a Reactive Medium. Patent US 20110052456 A1, 2011.

(19) Komorowska-Durka, M.; Barmen't Loo, M.; Sturm, G. S. J.; Radoiu, M.; Oudshoornc, M.; Van Gerven, T.; Stankiewicz, A. I.; Stefanidis, G. D. Chem. Eng. Process. 2013, 69, 83-89. 